\title{
V/Q scintigraphy: alive, well and equal to the challenge of CT angiography
}

\author{
Leonard M. Freeman • Linda B. Haramati
}

Published online: 30 January 2009

(C) Springer-Verlag 2009

It has been almost two decades since the Prospective Investigation of Pulmonary Embolism Diagnosis (PIOPED) study was published [1], yet its conclusions remain controversial $[2,3]$. Miscalculations in the design of the study, including the prospective parameters, can be attributed to the relatively limited fund of knowledge available to the creators of the PIOPED investigation. Within 3 years, modified criteria were retrospectively developed that, when applied to the PIOPED populations, provided more accurate results [4]. The enormous information that we have gained through the multiple retrospective analyses of the PIOPED data [5-10] are less well recognized than the original publication, although they have led to improvements in both the performance and the interpretation of $\mathrm{V} / \mathrm{Q}$ scintigraphy. After 20 years, it is time to stop critiquing this invaluable study and move ahead with the greater knowledge and more sophisticated approach that we have developed toward V/Q scintigraphy.

One of the major PIOPED critiques deals with the large number (44\%) of intermediate/indeterminate interpretations. We believe this is related to the fact that $68 \%$ of the study population comprised inpatients who are more likely

This Editorial Commentary refers to the article doi:10.1007/s00259008-1014-8.

L. M. Freeman $(\bowtie)$

Department of Nuclear Medicine,

Montefiore Medical Center and the Albert Einstein

College of Medicine,

Bronx, New York, NY, USA

e-mail: 1freeman@montefiore.org

L. M. Freeman • L. B. Haramati

Department of Diagnostic Radiology,

Montefiore Medical Center and the Albert Einstein

College of Medicine,

Bronx, New York, NY, USA to have underlying cardiopulmonary disease, such as pneumonia, chronic obstructive lung disease and pleural effusions that will cause "triple matches", resulting in intermediate or indeterminate interpretations. At Montefiore Medical Center, the great majority of our V/Q studies are performed in relatively young emergency department patients (average age in 2007 was 50.8 years, compared to 56.7 years for CT angiography) without these underlying conditions. We generally screen patients with chest radiography which, when normal or near-normal, can be followed with a very interpretable V/Q study. This was one of the important observations made from retrospective PIOPED analysis and described by the investigators and others $[8$, 11]. Our number of indeterminate interpretations in more than 2000 studies performed in 2006 and 2007 was 6.5\%. Additionally, correlating the indeterminate studies with clinical findings and pretest probability often allows us to skew the interpretation to the low or high portion of the intermediate category making it clinically more useful.

Shortly following the original PIOPED study, helical computed tomographic angiography (CTA) was introduced [12]. As additional refinements, including multirow detectors, were developed, CTA overtook V/Q scintigraphy as the most commonly performed imaging modality for suspected pulmonary embolism (PE) [13]. This advanced technology has allowed greater detection of subsegmental PE [14]. Interestingly and importantly, the benefit of diagnosing more PE is uncertain as the risk of recurrent thromboembolism and deaths have not declined [15]. An advantage of CTA is its ability to make alternative diagnoses, e.g., aortic dissection or pneumonia that may explain the patient's symptoms $[14,16]$.

Magnetic resonance angiography (MRA) has been studied for nearly two decades, but has not yet found a routine role in the imaging of patients with suspected PE. 
The PIOPED III study was designed to study the accuracy of gadolinium-enhanced MRA in combination with venous phase magnetic resonance venography for the diagnosis of acute PE. Although recently completed, the results are not yet available. However, the investigators have published an article describing the methods used in the study [17].

Currently, debate remains regarding the appropriateness of performing V/Q scintigraphy or CTA as the initial imaging procedure for suspected PE. In an accompanying article in this issue of the EJNM, an IAEA review addresses the relevant facts and controversies [18]. In general, we agree with the conclusions and recommendations presented in this excellent and thorough document which should be carefully read in its entirety. Several issues discussed in this review are of particular importance and merit further analysis.

\section{Clinical assessment and the role of pretest probability (PTP)}

There is general agreement that the clinical diagnosis of PE is unreliable as several other cardiopulmonary disorders will present similarly $[19,20]$. The clinician's assessment of pretest probability is, however, considered relevant. PIOPED II, which primarily studied the accuracy of CTA, showed a poor positive predictive value (PPV) of only $58 \%$ when the CTA results and pretest probability were discordant [21]. Interestingly, a similar poor performance (PPV of 56\%) was noted when V/Q scintigraphy results were discordant with pretest probability in PIOPED I [1]. A conclusion of PIOPED II was that when results of imaging are discordant with pretest probability, further investigation is needed. It is reasonable to suggest that when imaging results for either V/Q scintigraphy or CTA are discordant with the pretest probability, the alternative test should be recommended.

The IAEA review refers to three available scoring systems to objectively judge the pretest probability of PE. These are the Hamilton (Wells), PISA and Geneva systems. The Wells classification [22] and its modification [23] have achieved the greatest popularity. However, these systems are used by a minority of clinicians. As described in a survey conducted by a team at Johns Hopkins, $72.5 \%$ of clinicians prefer their own, subjective, assessment [24]. The use of an objective assessment is preferred since it provides a richer dataset for residents and can be taught to junior clinicians in hours rather than the decades it may take to acquire accurate "subjective judgment".

\section{Relative accuracy of V/Q scintigraphy and CTA}

PIOPED II focused on the accuracy of CTA rather than comparing its accuracy with V/Q imaging [21]. In fact, the
V/Q scan actually represented the most frequently used reference standard required for entry into the study. The overall sensitivity and specificity of CTA in the 824 patients studied was $83 \%$ and $96 \%$ respectively, after $6 \%$ (51 patients) of the study population were removed for technical inadequacy. With the entire study population included, the overall sensitivity and specificity of CTA declined to $78 \%$ and $90 \%$, respectively. The overall PPV of $86 \%$ and negative predictive value (NPV) of $95 \%$ are values comparable to V/Q statistics. The performance of V/Q studies has been enhanced by improved instrumentation and the use of a large number of ancillary scintigraphic findings, many of which resulted from retrospective review of PIOPED I data. These have been reviewed in detail [10] and are summarized in Table 1 which recently appeared in a review in Seminars in Nuclear Medicine [25].

One of the major parameters in judging the effectiveness of a diagnostic procedure is examining its rate of false negatives (FN). In patients with suspected PE and negative imaging, a subsequent diagnosis of PE or deep vein thrombosis (DVT) within 3 months constitutes a reasonable FN. Results from two recent studies support the comparable FN rate of V/Q scintigraphy and CTA. In a large, prospective randomized Canadian study in more than 1,400 patients with high pretest probability and/or positive D-dimer levels, the FN rates for V/Q scintigraphy and CTA were similar at $1 \%$ and $0.4 \%$, respectively [26]. In a study conducted at our institution in over 2,000 patients, the FN rates were statistically equivalent at $1.1 \%$ for $\mathrm{V} / \mathrm{Q}$ scans and $1.2 \%$ for CTA [27] when the chest radiograph was used to guide the choice of procedure.

\section{Is perfusion imaging alone adequate?}

The Prospective Investigation Study of Acute Pulmonary Embolism (PISAPED) proposed using a combination of pretest probability, chest radiography findings and perfusion scan only to evaluate patients with suspected PE [28]. These investigators felt that the finding of wedge-shaped defects on the perfusion scan can make a diagnosis of PE "irrespective of the radiographic findings in the corresponding lung regions" [29]. Radiographic findings such as oligemia and consolidation suggesting infarction help when they can be distinguished from emphysema, congestive failure and more typical pneumonic consolidations. The investigators state that the chest radiograph is not to be used as a surrogate for the ventilation scan. Two recent articles by the proponents of this PISAPED methodology claim an $85 \%$ PPV and $96 \%$ NPV which, when retrospectively applied to the PIOPED II patient population $[29,30]$, are comparable to the CTA values in the same study ( $86 \%$ PPV, 95\% NPV). The V/Q scan data using modified PIOPED criteria were $72.4 \%$ PPV and $96.5 \%$ NPV and the number of nondiagnostic studies fell to zero. 
Table 1 Improvements in V/Q methodology and interpretation since PIOPED I (modified from reference [25])

1. The original PIOPED study had a very heavy concentration on inpatients, who constituted $68 \%$ of the total population studies. PIOPED II had an inpatient population of $11 \%$. Inpatients are much more likely to have abnormalities on chest radiographs that would potentially interfere with optimal V/Q scan interpretation. Screening patients by chest radiography has very significantly cut down the number of intermediate, nondiagnostic interpretations.

2. The use of a number of ancillary scintigraphic findings not used in PIOPED I subsequently became available to us. Some of these were based on data made available from retrospective review of PIOPED. Most of these allow a very low probability or PE ABSENT interpretation. These include:

a. The stripe sign

b. The fissure sign

c. Segmental contour pattern

d. Large pleural effusions with matched V/Q scintigraphy findings and no other V/Q scan mismatches

e. Radiographic densities with matched V/Q scintigraphy findings in upper or mid-lung zone

f. Perfusion scan better than abnormal chest radiograph

g. V defects worse than Q defects: reverse mismatch

3. Stratification of patients who may or may not have underlying cardiopulmonary disease has enhanced interpretation.

4. Retrospective analysis of the PIOPED criteria found errors, e.g., a moderate single segmental mismatch was erroneously called low probability. In a subsequent publication modifying the original criteria, the single segmental mismatch was correctly placed in the intermediate category.

5. Different significance of findings when correlated with objective clinical assessment (pretest probability), i.e., a single segmental mismatch in a patient with high pretest probability constitutes a high probability V/Q scan interpretation.

6. Improved particle ventilation agents are now available that can be used in place of the older, but still superb xenon-133 study. The optimal particle, inhalatory agent, Technegas (Cyclopharm), has been used worldwide outside the US for the past 15 years. It will, hopefully, receive approval from the Food and Drug Administration within the next year.

7. Nuclear medicine instrumentation has improved considerably. Most centers use dual headed detectors to considerably shorten the time of the examination. Those that continue to use single headed cameras have instruments with significantly better resolution than those used in PIOPED I. Very few of the cameras used in the mid-1980s for PIOPED I would be acceptable by today's standards.

In addition, the use of SPECT in many centers, primarily outside the US, has improved diagnosis.

As concluded by the authors, the use of the PISAPED interpretive scheme is of greatest value when used by individuals or a closely integrated team with expertise in clinical evaluation, as well as radiographic and scintigraphic interpretation. Since this type of universal expertise is not always available, the continued use of the ventilation scan is a safer, justifiable approach in most medical centers, although its elimination would reduce cost and radiation exposure. The
PISAPED scintigraphic criteria place enormous weight on whether a perfusion defect is wedge-shaped, which is not always simple to characterize on planar images. Accordingly, there has been one study that advocates a perfusion-only SPECT study as performing better than perfusion-only planar studies and eliminating most "nondiagnostic" or intermediate studies [31].

One area where a perfusion-only study works well is in pregnant patients suspected of having PE. The IAEA article recommends a low dose (approximately $40 \mathrm{MBq}$ of ${ }^{99 \mathrm{~m}} \mathrm{Tc}$ MAA) perfusion-only study [18]. We agree completely and have used this approach for the past two decades. In our experience, more than $95 \%$ of these examinations have been normal or near-normal.

\section{The radiation issue}

Much has been written, particularly in the past 2 years, about the high radiation exposure, particularly of the female breast, that is associated with CTA [32-34]. The estimate of 20$60 \mathrm{mSv}$ is considerably higher than the $0.28-0.9 \mathrm{mSv}$ estimate for V/Q scintigraphy [35]. Another interesting comparison can be made to a standard two-view mammogram which delivers approximately $3 \mathrm{mSv}[36]$. The 10-20 times greater exposure from CTA is equivalent to a life-time of mammograms. Einstein et al. [33] also project an increase in life-time breast cancer risk of 1 in 143 for a 20-year-old woman and 1 in 284 for a 40-year-old woman who undergoes ECG-gated CTA. As pointed out in an American College of Radiology white paper [37], it is our responsibility as imaging physicians to be thoroughly familiar with the radiation risks associated with each procedure and in turn, educate the referring physician. V/Q scintigraphy and CTA represent an excellent example of diagnostically equivalent procedures where chest radiography results can help guide the choice of modality.

\section{The "after hours" availability issue}

A physician in a busy emergency department generally requires a timely diagnosis for a patient suspected of having a PE. Most medical centers have CT scanners with an onsite technologist available at all times. As a result, many physicians favor CTA over V/Q scanning, even in the setting of a negative chest radiograph. In our institution, a nuclear medicine technologist is on the premises until midnight and is available on-call thereafter. The technologist is able to reach the hospital, prepare the radiopharmaceuticals and complete the study within 1.5 to 2 hours. This time-frame is acceptable to our clinicians for the vast majority of stable patients. If the patient is hemodynamically unstable, CTA is the modality of choice. 
If after-hours imaging is not available and a patient has a high clinical suspicion of PE, a reasonable approach includes administering a single dose of low molecular weight heparin and imaging the patient the next morning. This strategy may be particularly advisable for performing V/Q scintigraphy in young women to avoid the excessive breast radiation exposure associated with CTA.

\section{Competence in interpreting $V / Q$ scans}

The shift to performing CTA has resulted in a lack of comfort of imaging physicians in appropriate interpretation of $\mathrm{V} / \mathrm{Q}$ scans. Radiology and Nuclear Medicine residents in many centers do not receive adequate training in interpreting V/Q scans. It is our responsibility to ensure that all residents interpreting "after hours" V/Q studies are as comfortable with V/Q scan as with CTA interpretation. Toward this end we have created a computer-based training program and require each resident to interpret 20-25 typical and atypical V/Q studies as a structured component of their training.

\section{Role of V/Q scintigraphy as an important baseline examination}

There are two major areas where V/Q scans are important as a monitoring tool. Even if the diagnosis of PE is made with CTA, it is useful to perform a baseline V/Q scan with follow-up studies at 1 week, 1 month and 3 months until complete resolution or stability. Significant resolution beyond 3 months is unlikely [38]. If symptoms recur, it will be possible to distinguish a new from an old PE. This approach avoids the high radiation exposure associated with multiple repeat CTA scans.

The second clinical scenario is in patients being anticoagulated for DVT. Since silent PE are known to occur in $38-50 \%$ of patients with DVT $[39,40]$, a baseline study at the start of anticoagulant therapy is quite useful. If the patient subsequently develops symptoms of $\mathrm{PE}$, it will then be possible to determine if the PE occurred before or after initiation of anticoagulation. Without baseline imaging, it may be impossible to determine whether the PE was part of the initial presentation of thromboembolic disease or whether it represents a failure of anticoagulation, perhaps requiring placement of an inferior vena cava filter.

\section{The language of lung scan interpretation}

Probability terminology has created confusion amongst both clinicians [41] and imaging physicians [42]. This particularly applies to the low probability category. Biello's original retrospective classification [43] assigned a $<10 \%$ PPV to this category which PIOPED changed to an unacceptable $<20 \%$ PPV. To rectify this, Gottschalk et al. described a very low probability category with a return to a $<10 \%$ PPV [44]. We propose that V/Q scintigraphy be interpreted as either "positive for PE", "nondiagnostic study" or "no evidence of PE". This will make it more understandable for clinicians and avoid the confusion associated with probability interpretations. This suggested classification was used in the Anderson Canadian Study where their very low FN rates $(1.0 \%$ for $\mathrm{V} / \mathrm{Q}$ scintigraphy and $0.4 \%$ for CTA) for both procedures validate its use [26]. The PEs missed on a "no evidence of PE" interpretation are very likely to be of the small subsegmental variety that apparently do not require anticoagulant treatment (see following section). There is one note of caution regarding the use of this binary (yes/no) system of interpretation. As both PIOPED studies showed, discordance between clinical likelihood and either V/Q scintigraphy or CTA results are relatively inaccurate and often require further testing $[1,21]$.

\section{The dilemma of small PE and whether they require treatment}

Anderson et al. showed that more emboli were detected by CTA than by V/Q scintigraphy [26]. In the concluding sentence of the report of their study as well as in an accompanying editorial [45] the clinical significance of detecting and treating these smaller emboli was questioned. The importance of enhanced detection of PE by CTA remains an important question [46-48] as the risk of recurrent $\mathrm{PE}$ and death have not decreased during the CTA era [15].

Although not proven, it is believed that the pulmonary capillary bed traps small emboli and prevents them from entering the systemic circulation, possibly even in normal individuals [47]. The IAEA article references a 1994 study [49] in which patients with DVT were randomized to either anticoagulation or a nonsteroidal antiinflammatory agent. In each group, 50\% developed PE. They concluded that anticoagulant therapy had no effect on disease progression.

The burden of clot has prognostic significance and may be a major determinant of whether anticoagulant therapy is appropriate. Patients with underlying cardiopulmonary disease who develop even small PE are at greater risk of developing right heart failure, death and chronic pulmonary hypertension [50] and should likely be anticoagulated. We sometimes are asked to perform V/Q studies in pulmonary hypertension patients to evaluate for chronic PE. If present, chronic $\mathrm{PE}$ is treated with anticoagulation to prevent disease progression [51]. 
Goodman recently has defined several patient groups where the risks of anticoagulant therapy may outweigh the benefits [47, 52]. Clearly, further prospective, controlled studies are needed to resolve this problem. In this era of economic hardship and enormous pressures on health-care systems worldwide, practicing outcomes-based medicine has become a paramount goal.

\section{The issue of planar vs. SPECT V/Q scintigraphy}

Over the past several years, the advantages of performing SPECT rather than planar V/Q scintigraphy [53-55] have been detailed. These reports all originate outside the US. Many of our close and valued colleagues question why we continue to perform planar imaging instead of using the more modern SPECT technology [2,3]. Although we are not opposed to using SPECT, our results using planar imaging are excellent and we do not yet possess all of the tools needed to perform state-of-the-art SPECT V/Q studies. As described recently by Roach et al. [55], a superior ventilation agent is required to adequately support SPECT methodology. Technegas (Cyclopharm, Sydney, Australia) best fulfills this requirement. It has been available outside the US for more than a decade, but has yet to be approved by the American Food and Drug Administration. Although a few American colleagues have switched to SPECT with available agents, we prefer to wait for Technegas.

We have done well with planar imaging with a FN rate of only $1.1 \%$ comparable to the $1.0 \%$ rate described by Anderson et al. [26]. Using SPECT, Leblanc et al. [56] reported a NPV of $98.5 \%$, which is better than historical results of planar imaging, but comparable to the results of planar imaging using current technology [25-27].

If additional PE are demonstrated on SPECT, it is not clear that treating these smaller PE confers a benefit in terms of outcome. Hence, the major advantage of SPECT is not necessarily the detection of smaller PE; rather, it is the ease of interpreting SPECT studies. In our institutions, we have 32 residents interpreting "after hours" lung scans. We spend a lot of time and effort teaching them how to do this properly. A recent monitoring survey showed a less than $1 \%$ discordance between resident and staff physician readings which generally are made several hours later. If SPECT makes interpretation easier, we will gladly embrace its use as soon as Technegas becomes available in the US.

In conclusion, competition and controversy exists between two superb diagnosis modalities, V/Q scintigraphy and CTA, for imaging patients with suspected PE. Although studies show equivalent outcomes-based results, the ready availability of CTA, particularly after hours, and the bias towards anatomic rather than functional imaging has made
CTA the initial imaging modality in most institutions. Excess radiation exposure is a major drawback of CTA. It is the responsibility of imaging physicians and basic scientists to educate clinicians about the radiation burden associated with each procedure and recommend protocols to minimize it. We propose the use of chest radiography as a triaging tool to determine which study to recommend. SPECT may further enhance the diagnostic accuracy of V/Q scintigraphy and validate its role as the primary imaging study in many patients with suspected PE.

\section{References}

1. The PIOPED Investigators. Value of the ventilation/perfusion scan in acute pulmonary embolism. Results of the prospective investigation of pulmonary embolism diagnosis (PIOPED). JAMA 1990;263:2753-9.

2. Reinartz P. To PIOPED or not to PIOPED. J Nucl Med 2008;49: $1739-40$.

3. Uren R. Letter to the editor. J Nucl Med (in press for April 2009).

4. Gottschalk A, Sostman HD, Juni JE, et al. Ventilation-perfusion scintigraphy in the PIOPED study. Evaluation of the scintigraphic criteria and interpretation. J Nucl Med 1993;34:1119-26.

5. Sostman HD, Gottschalk A. Prospective validation of the stripe sign in ventilation-perfusion scintigraphy. Radiology 1992;184: $455-9$.

6. Worsley DF, Kim CK, Alavi A, et al. Detailed analysis of patients with matched ventilation-perfusion defects and chest radiographic opacities. J Nucl Med 1993;34:1851-3.

7. Kim CK, Worsley DF, Alavi A. Ventilation-perfusion-chest radiography: match is less likely to represent pulmonary embolism if perfusion is decreased rather than absent. Clin Nucl Med 2000; 25:665-9.

8. Stein PD, Gottschalk A, Henry JW, et al. Stratification of patients according to prior cardiopulmonary disease and probability assessment based on the number of mismatched segmental equivalents. Chest 1993;104:1461-7.

9. Stein PD, Gottschalk A. The addition of clinical assessment to stratification according to prior cardiopulmonary disease further optimizes the interpretation of ventilation-perfusion lung scans in pulmonary embolism. Chest 1993;104:1472-6.

10. Freeman LM, Krynyckyi B, Zuckier LS. Enhanced lung scan diagnosis of pulmonary embolism with the use of ancillary scintigraphic findings and clinical correlation. Semin Nucl Med 2001;31:143-57.

11. Forbes KP, Reid JH, Murchison JT. Do preliminary chest x-ray findings define the optimum role of pulmonary scintigraphy in suspected pulmonary embolism? Clin Radiol 2001;56:397-400.

12. Remy-Jardin M, Remy J, Wattinne L, et al. Central pulmonary thromboembolism diagnosis with spiral, volumetric CT with the single breath hold technique - comparison with pulmonary angiography. Radiology 1992;185:391-7.

13. Stein PD, Kayalli F, Olson RE. Trends in the use of diagnostic imaging in patients hospitalized with acute pulmonary embolism. Am J Cardiol 2004;93:1316-7.

14. Schoepf UJ, Holzknecht N, Helmberger TK, et al. Subsegmental pulmonary emboli: improved detection with thin collimation multi-detector row spiral CT. Radiology 2002;22:483-90.

15. Burge AJ, Freeman KD, Klapper PJ, et al. Increased diagnosis of pulmonary embolism without a corresponding decline in mortality during the CT era. Clin Radiol 2008;63:381-6. 
16. Cronin P, Weg JG, Kazerooni EA. The role of multi-detector computed tomography angiography for the diagnosis of pulmonary embolism. Semin Nucl Med 2008;38:418-31.

17. Stein PD, Gottschalk A, Sostman HD, et al. Methods of prospective investigation of pulmonary embolism diagnosis III. Semin Nucl Med 2008;38:462-70.

18. Reid JH, Coche EE, Inoue T, et al. Is the lung scan alive and well? Facts and controversies in defining the role of lung scintigraphy for the diagnosis of pulmonary embolism in the era of MDCT. Eur J Nucl Med Mol Imaging. doi:10.1007/s00259-008-1014-8.

19. Stein PD, Terrin ML, Hales CA, et al. Clinical, laboratory, roentgenographic and electrocardiographic findings in patients with acute pulmonary embolism and no pre-existing cardiac or pulmonary disease. Chest 1991;100:598-603.

20. Goldhaber SZ. Pulmonary embolism. Lancet 2004;363:1295-305.

21. Stein PD, Fowler SE, Goodman LR, et al. Multi-detector computed tomography for acute pulmonary embolism. N Engl J Med 2006;354:2317-27.

22. Wells PS, Anderson DR, Rodger MA, et al. Excluding pulmonary embolism at the bedside without diagnostic imaging. Management of patients with suspected pulmonary embolism presenting to the emergency department by using a simple clinical model and Ddimer. Ann Intern Med 2001;135:98-107.

23. Van Belle A, Buller HR, Hussman MV; Christopher Study Investigators. Effectiveness of managing suspected pulmonary embolism using an algorithm combining clinical probability, Ddimer testing and computed tomography. JAMA 2006;295:172-9.

24. Weiss CR, Haponik EF, Giette GB, et al. Pretest risk assessment in suspected acute pulmonary embolism. Acad Radiol 2008;15:3-14.

25. Freeman LM, Stein EG, Sprayregen S, et al. The current and continuing important role of ventilation-perfusion scintigraphy in evaluating patients with suspected pulmonary embolism. Semin Nucl Med 2008;38:432-40.

26. Anderson DR, Kuhn SR, Rodger MA, et al. Computed tomographic pulmonary angiography vs. ventilation-perfusion lung scanning in patients with suspected pulmonary embolism. A randomized controlled trial. JAMA 2007;298:2743-53.

27. Stein EG, Haramati L, Chamarthy M, et al. Success of a simple and safe algorithm to reduce utilization of CT pulmonary angiography in the emergency department. Accepted for presentation at the American Roentgen Ray Society, April 2009, Boston, MA.

28. Miniati M, Pistolesi M, Marini C, et al. Value of perfusion lung scan in the diagnosis of pulmonary embolism: results of the prospective investigative study of acute pulmonary embolism (PISA-PED). Am J Res Crit Care Med 1996;154:1387-93.

29. Miniati M, Sostman HD, Gottschalk A, et al. Perfusion lung scintigraphy for the diagnosis of pulmonary embolism: a reappraisal and review of the prospective investigative study of pulmonary embolism diagnosis methods. Semin Nucl Med 2008;38:450-61.

30. Sostman HD, Miniati M, Gottschalk A, et al. Sensitivity and specificity of perfusion scintigraphy combined with chest radiography for acute pulmonary embolism in PIOPED II. J Nucl Med 2008;49:1741-8.

31. Collart JP, Roelants V, Vanpee D, et al. Is a lung perfusion scan obtained by using single photon emission computed tomography able to improve the radionuclide diagnosis of pulmonary embolism? Nucl Med Commun 2002;23:1107-13.

32. Brenner DJ, Hall EJ. Computed tomography an increasing source of radiation exposure. N Engl J Med 2007;357:2277-84.

33. Einstein AL, Henzlova MJ, Rajagopolan S. Estimating risk of cancer associated with radiation exposure from 64 slice computed tomography coronary angiography. JAMA 2007;298:317-23.

34. Task Group on Control of Radiation Dose in Computed Tomography. A report of the international commission on radiologic protection. Ann ICRP 2000;30:7-35.
35. Radiation Dose to Patients From Radiopharmaceuticals. Ann ICRP 1998;28:1-126.

36. Parker MS, Hui FK, Camacho MA. Female breast radiation exposure during CT pulmonary angiography. AJR Am J Roentgenol 2005; 185:1228-31.

37. Amis ES, Butler PF, Applegate KE, et al. American College of Radiology white paper on radiation dose in medicine. J Am Coll Radiol 2007;4:272-84.

38. Wartski M, Collignon MA. Incomplete recovery of lung perfusion after 3 months in patients with acute pulmonary embolism treated with antithrombotic agents. THESEE Study Group. Tinzaparin ou Heparin Standard: Evaluation dans l'Embolie Pulmonaire Study. J Nucl Med 2000;41:1043-8.

39. Moser KM, Fedullo PF, Littlejohn JK, et al. Frequent asymptomatic pulmonary embolism in patients with deep venous thrombosis. JAMA 1994;271:223-5.

40. Monreal M, Ruiz J, Olazabaz A, et al. Deep venous thrombosis and the risk of pulmonary embolism. Chest 1992;102:677-81.

41. Gray HW, McKillup JH, Besent RG. Lung scan reports: interpretation by clinicians. Nucl Med Commun 1993;14:989-94.

42. Gray HW, McKillup JH, Besent RG. Lung scan reporting language. What does it mean? Nucl Med Commun 1993;14: 1084-7.

43. Biello DR, Mattar AJ, McKnight RC, et al. Ventilation-perfusion studies in suspected pulmonary embolism. AJR Am J Roentgenol 1979; 133:1033-7.

44. Gottschalk A, Stein PD, Sostman HD. Very low probability interpretation of ventilation-perfusion lung scans in combination with low probability clinical assessment reliably excludes pulmonary embolism: data from PIOPED II. J Nucl Med 2007;48:1411-5.

45. Glassroth J. Imaging of pulmonary embolism: too much of a good thing. JAMA 2007;298:2788-9.

46. Noveline R, Balatonowich O, Athanosoulis C, et al. The clinical course of patients with suspected pulmonary embolism and a negative pulmonary angiogram. Radiology 1978;126:561-7.

47. Goodman L. Small pulmonary emboli. What do we know? Radiology 2005;234:654-8.

48. Desai SR. Unsuspected pulmonary embolism on CT scanning: yet another headache for clinicians? Thorax 2007;62:470-2.

49. Nielsen HK, Husted SE, Krusell LR, et al. Silent pulmonary embolism in patients with deep venous thrombosis: incidence and fate in a randomized, controlled trial of anticoagulation vs. no anticoagulation. J Intern Med 1994;235:457-61.

50. Oser RF, Zuckerman DA, Gutierrez FR, et al. Anatomic distribution of pulmonary emboli at pulmonary angiography: implications for cross-sectional imaging. Radiology 1996;191:31-5.

51. Fishman AJ, Moser KM, Fedullo PF. Perfusion lung scans vs pulmonary angiography in evaluation of suspected pulmonary hypertension. Chest 1983;84:673-83.

52. Eyer BA, Goodman LR, Washington L. Clinicians' response to radiologists' reports of isolated subsegmental pulmonary embolism or inconclusive interpretation of pulmonary embolism using MDCT. AJR Am J Roentgenol 2005;184:623-8.

53. Reinartz P, Wilberger JE, Schaefer W, et al. Tomographic imaging in the diagnosis of pulmonary embolism. A comparison between V/Q lung scintigraphy in SPECT technique and multi-slice spiral CT. J Nucl Med 2004;45:1501-8.

54. Bajc M, Bitzen U, Olsson B, et al. Diagnostic evaluation of planar and tomographic ventilation/perfusion lung images in patients with suspected pulmonary emboli. Clin Physiol Funct Imaging 2004;24:249-56.

55. Roach PJ, Bailey DL, Schembri GP. Reinventing ventilation/perfusion lung scanning with SPECT. Nucl Med Commun 2008;29:1023-5.

56. Leblanc M, Leveillee F, Turcotte F. Prospective evaluation of the negative predictive value of V/Q SPECT using $99 \mathrm{mTc}-T e c h n e g a s$. Nucl Med Commun 2007;28:667-72. 